\title{
Research of virtual substation simulation training system
}

\author{
Wu Xu, Xitong Duan ${ }^{\mathrm{a}}$, Yilong Ren, Lei Wu and Jin He \\ Yunnan Minzu University, Kunming, Yunnan 650500,China
}

\begin{abstract}
Firstly, this paper analyzes the current status of substation training system, and puts forward a substation simulation training system based on virtual reality technology, aiming at the shortcomings of traditional substation training system. The system bases on the building of substation model, introducing platform VIZARD, using MATLAB simulation data, by COM technology in VIZARD of data simulation system call, also using wearable devices and finally realizes virtual substation simulation training system. The system overcomes the shortcomings of the low degree of immersion and low truth degree, which is more economical and safe.
\end{abstract}

Keywords: virtual reality; digital substation; VIZARD; human-computer interaction.

\section{Introduction}

In twenty-first Century, China's power grid construction has entered a new stage of rapid development, the construction of power grids to ensure sustained and stable development of national economy. The development of smart grid in China is the "12th Five-Year" stage to today's "13th Five-Year" planning under the inevitable choice of social and economic development. As an important part of power system, the safe operation of the substation directly affects the stable operation of the power grid, which is an important factor to ensure the reliable power supply of the power grid [1]. With the development of science and technology, the new generation of power system's devices will gradually eliminate obsolete and outdates equipment, these new equipment will improve the degree of automation, reliability, information. Then, on relevant operation personnel comprehensive quality also put forward a higher requirements, how to cultivate the master so that a new generation of substation operation technology of personnel has become an urgent problem to be solved, and the problem is also related to the power grid can stable operation [2]. Therefore, to improve the technical level of the substation operation, to make sure their safe and efficient, we should use this training system to improve the degree of automation of substation.

At present, domestic substation training system is traditional visit teaching mode like disk desktop and full software training. Traditional visit mode has many disadvantages such as poor teaching security, reel model covers a large space, high expense, low training efficiency, update replacement ability is weak; software training system and the training efficiency are quiet insufficient, authenticity is obviously out of date. [3][4]. In view of the situations above, the substation simulation training system based on virtual reality technology arises at the historic moment [5]. Virtual reality,also known as VRrefers to the people use the computer to calculate complex data to construct a new pattern of visualization and interaction. It can use computer to create realistic 3D auditory, visual, tactile,

${ }^{\mathrm{a} C}$ Corresponding author : duanxitong25@163.com

(C) 2016. The authors - Published by Atlantis Press 
olfactory sensation. Let users operate special input and output devices, naturally have virtual world experience, resulting in immersive feel [6]. This technology has the characteristics of high immersion, interactivity, economy and security [7]. Therefore, the use of Virtual Substation Simulation and training system can overcome the low efficiency of the traditional training mode, poor security, insufficient of reliability. To achieve the hazardous environment training of substation visual, the prospect of real security training application is very broad [8].

\section{Design of virtual substation simulation training system}

With help of measuring actual substation, we can model parameters of the corresponding substation equipment to build the prototype system. And then the high definition digital photography, scanning images, maps and other means use 3DMAX technology and three-dimensional technique to model substation,and get into VIZARD model for each device structure. Finally establish a realistic 3D substation model. The construction of data simulation system in MATLAB, and the dynamic link with VIZARD software platform constitute our simulation operating system. The specific process is shown in figure1:

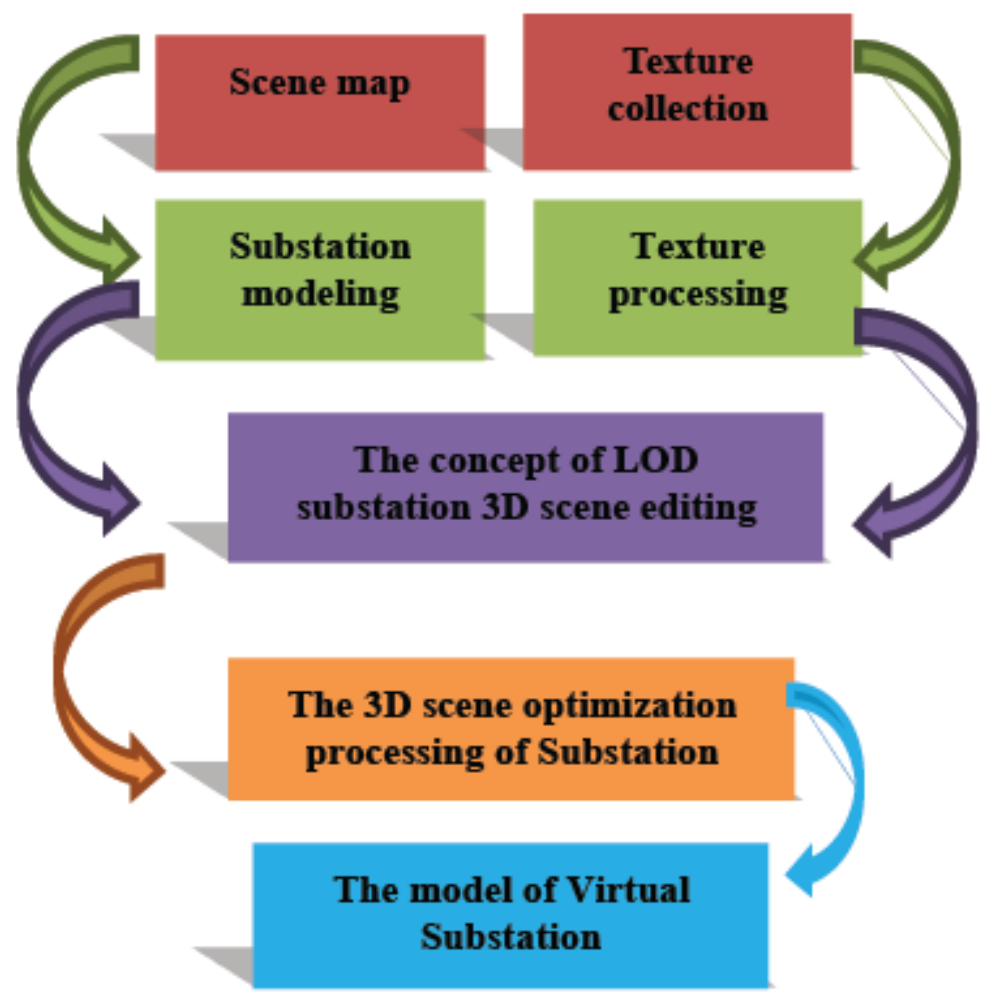

Figure 1.Model Build Flow chart

\subsection{Modeling of virtual substation}

\subsubsection{Model building}

Creating the Virtual Substation mainly use geometric model and image model, which are supported by a variety of virtual model and analysis of virtual data. The Virtual Substation Scene is overall designed construction, mainly includes module of substation equipment, environment module and module for surface features. 
Geometry modeling is mainly about the geometric information of objects. Geometric modeling technology based on the real scene. Firstly it should be fuzzy and abstracted, and then create a good outline of the geometry of the substation. Finally use the three-dimensional modeling software 3DMAX to create. But in the virtual scene, it is likely that the picture is not stable, which causes the decrease of the environmental reality, which is only suitable for the construction of a simple model. Because of the complex structure of the equipment, we used image modeling technology, through the camera shooting image or a 3D scanning equipment acquisition equipment basic information. Shooting or 3D scanning device seamless splicing can generate graph model, the spatial association set and spatial operation capability of realistic scenarios.

\subsubsection{Model optimization}

LOD (levels of detail technology concept) whose object covers a smaller area of the screen, uses the object model to describe the coarser and gives a decision algorithm in the geometric level model for complex scenes which is fast rendering by [9]. The details of the model are simplified by using the specific rules. The concept mainly simplifies several parts of contents: firstly, do not need to use graphics hardware rendering details; secondly, cannot use graphics hardware rendering details, such as methods based on distance and object size standard; thirdly, people observed by naked eye to details.

Using the LOD concept, and using texture processing method reasonably, not only will not affect the visual effect and interaction of virtual scene in substation, but also greatly improve the speed of the CPU operation so that the scene can be accelerated.

\subsubsection{Combination of Virtual Substation model and simulation system}

VIZARD software platform is based on $\mathrm{C} / \mathrm{C}++$, using the new OpenGL development module developed by the high performance graphics engine. VIZARD platform supports a lot of 3D format, such as.wrl,.flt,.3ds,.obj,.pfb, etc.. So we will use 3DMAX to build the Virtual Substation model using.3ds file formats to store, the export and import of this file format is very convenience. At the same time, it can solve the problems in a large database of 3D model so that they can be used directly in the VIZARD platform.

\subsection{Database simulation}

In the substation simulation training system, a large amount of data is needed to support the development of modeling and simulation process in advance. Data acquisition, such as simulation model of basic parameters, simulation model initialization data, simulation analysis judgment data and simulation data and other information can carry on the simulation analysis of these data to ensure that the training of personnel in the validity of the virtual platform for the operation of the system response.

\subsubsection{Data simulation software and environment loading platform}

In the process of data simulation, the Systems SimPower module in MATLAB/Simulink is used to carry out the simulation of the normal operation of the Virtual Substation and the fault operation. The advantages are that the precision is high and the economy is convenient.

At the same time, VIZARD software is used for the environment loading platform by us. VIZARD software is the Virtual Reality development platform software, which is based on the C/C++, using the new OpenGL development module developed by the high performance graphics engine. The environmental platformuse Python language to load the three-dimensional virtual scene, control the three-dimensional virtual scene and some of the operation. 


\subsubsection{Simulation platform and VIZARD interaction}

MATLAB data simulation software use $C$ language and Fortran language to edit, and VIZARD use Python language to implement the development, so it cannot be called directly. We will use the COM technique to take the file of MATLAB into the VIZARD platform program[10]. MATLAB compiler generateCOM component and need to use external $\mathrm{C}++$ compiler, and then the COM component will be transformed to generate the $M$ file conversion by MATLAB software. And then transform the $M$ file to simulation DLL Dynamic link library.Next, we operate the VIZARD platform by using the corresponding Python language, adding thecalled-sentence of .dll Dynamic link library.Finally,it can be implemented in VIZARD platform of MATLAB simulation program data calls. The specific process is shown in figure2:

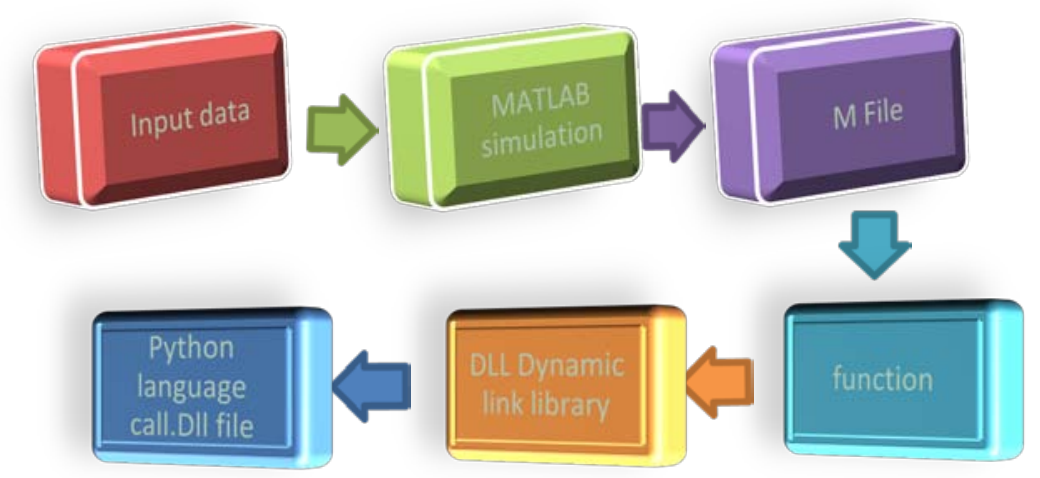

Figure 2. MATLABand VIZARD to achieve interactive flow chart

\section{Function realization of substation simulation training system}

The training content of a complete substation training system should be: Virtual Substation scene roaming, equipment operation control, fault processing training, assessment and evaluation. Main functions of the system are shown in figure 3 :

The training content is mainly realized by the following modules:

Roaming module: Using VIZARD software, our simulation uses the 3D helmets and special gloves of the 3D virtual digital substation to manipulate forward and backward movements, such as inspection and observation. Training personnel can use this inspection function of the Virtual Substation Scene and a variety of electrical equipment to get the first visual perception. But this mode is familiar with a variety of equipment connection modes. The module can leave the staffa roaming, realistic and objective feeling of the substation.

Data module: The use of MATLAB operating system is mainly to achieve a real-time simulation on the running state of substation under the real environment and to transmit the corresponding response to the platform database [11]. 


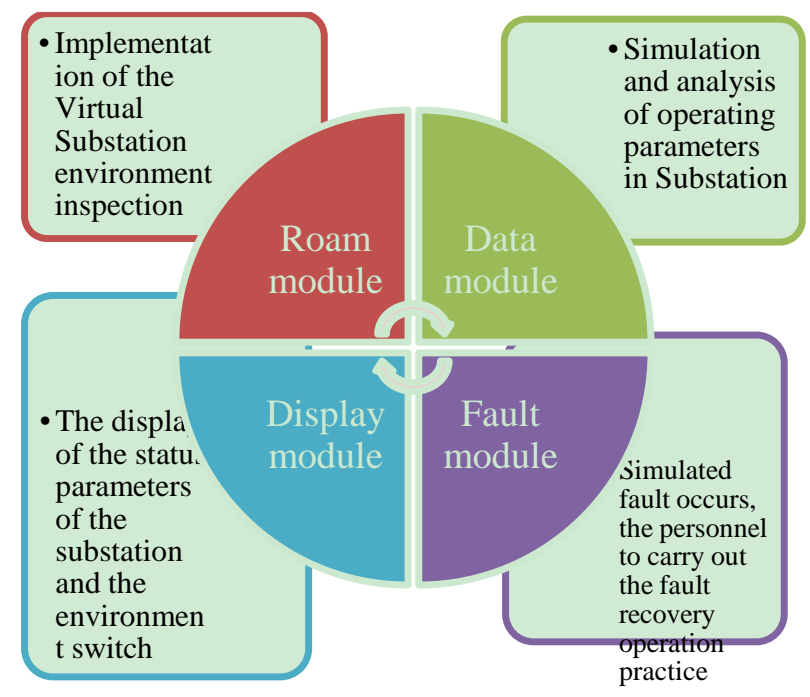

Figure 3. Main functions of the system

Display module: The establishment of a display module includes the status of the parameters of the display, the scene of the environment, such as switching scene. The system mainly realizes the human-computer interaction. In this module, personnel training uses data glove device switch. Turn the switch off triggers various substation responses which will go through the digital platform and 3D stereoscopic helmet. And then the intuitive performance produced by this module will leave a deep impression on the personnel.

Fault modules: When simulation fault appears, the power system will operate in a wrong way. With the help of this module, the personnel training can simulate the fault environment, and let the personnel give corresponding operation to solve the problem. At the same time, to achieve a variety of operations and visualization module interface, the correct operation and incorrect operation parameters change and state changes will be recorded by the visualization module.

Finally, with the help of these modules, we can achieve the training of personnel on the VIZARD platform.

\section{Human Computer Interaction System Design}

We use the substation simulation and training system to realize the real operation process. We use the 3D stereo helmet to bring training personnel into simulation environment of 3D for substation training to supervise. These devices can leave the personnel a high sense of substitution and authenticity. At the same time, with the help of special data gloves, the corresponding simulation operation and real human-computer interaction is accomplished. In virtual reality technology, data glove is an important virtual environment input and output equipment.The personnel can wear a data glove which is a special man-machine interface equipment to exchange data with computer. In the Virtual Substation environment of corresponding operation, the command will be received by staff immediately to achieve relatively ideal human-computer interaction.

During a specific data glove application process,the number of sensors is composed of a vector of parameter which is composed of each bending of the hand can be set up. Obviously there is a certain mapping relationship between $\mathrm{F}$ and $\mathrm{D}$.The data glove is set to find the inverse mapping of the original mapping relationship according to the vectors, so as to reverse the position of each part of the hand [12]. 
The purpose of the operation isto complete the instruction content which is called command gestures. The motion control transform can use gestures to express, and then the sensing device converts the input signal from the simulation to training system. The specific process is sfigure4:

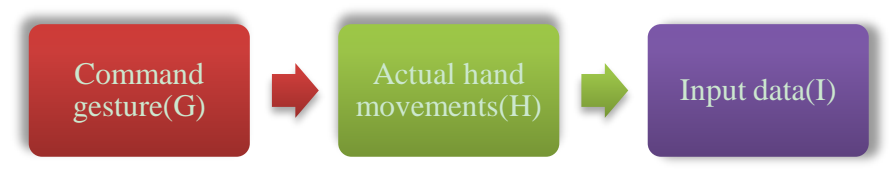

Figure 4. Gesture operation process diagram

$$
\begin{gathered}
\mathrm{T}_{\mathrm{gh}}: \mathrm{G} \rightarrow \mathrm{H}, \mathrm{H} \triangleq \mathrm{T}_{\mathrm{gh}} \\
\mathrm{T}_{\mathrm{hi}}: \mathrm{H} \rightarrow \mathrm{I}, \mathrm{I} \triangleq \mathrm{T}_{\mathrm{hi}}
\end{gathered}
$$

Among them, is for the human body motion control conversion function, and is for the sensing device conversion function. It can be seen that the realization of human-computer interaction in the process of gesture recognition, which is the system of gesture movement of the inverse process.

Training of personnel is intended by their hand movements convey to training system. At the same time, virtual simulation training system can translate the user gesture into operation command function. Finally, when personnel input different operating commands, computer based training system can execute commands through the different gestures and responds.

\section{Summary}

This paper is supported bythe virtual reality technology advanced technology concepts. This paper aims to build a Virtual Substation Simulation and training system. Thesystem uses the interaction platform of MATLAB and VIZARD, and realizes data and Virtual Substation environment interaction. Using a data glove to personnel training can directly send the control data to operate on the virtual substation, so that they can accurate simulation engaged in the work in the future. With the help of this training system,personnel can be familiar with the work environment in the future, and operate the complex and fault tolerance of the low rate of substation equipment. The system has the quality of high immersion and high mutual.It can also save the training system of hardware equipment investment, reduce the risk as possible as it can. The method of this system design can also be applied in military, medical, education and other fields.

\section{Acknowledgment}

This research is supported by the base of excellent talent training in Yunnan Province, project of virtual simulation experiment teaching center of electrical engineering in Yunnan Province and Regional science fund of the National Natural Science Foundation of China (Grant: 61365007/F030406).

\section{References}

1. Zhang Pu. "Substation Training System Based on Virtual Reality”. Tianjin University,2007.

2. Fang Shuyan. "Application and Development of Substation Training Simulation System”. High voltage technology. 2005, 31(4): 79-81.

3. Ding Ying, Ding Weidong, Han Dong. “500 kV Substation Simulation Training System”. Shandong Electric Power, 2000(2): 36-38.

4. Zhang Dongying, Ge Liang, Yang Yihan. "Realization of simulation and training system of $500 \mathrm{kV}$ integrated automation substation”. Power grid technology, 2001, 25(6): 64-66. 
5. Ye Peilei. “Analysis of virtual reality technology and Application”. Electronic technology and software engineering, 2015(9): 44.

6. $\mathrm{Xu} \mathrm{Wu}$, Gaofei. "Research of Virtual reality technology in Automotive engine Assembly teaching” the IEEE Joint International Information Technology and Artificial Intelligence Conference (ITAIC 2011). 2011,8(1). 167-169.

7. Lin Xiangyi. "Application of virtual reality technology in electric power training“. Northeast Petroleum University,2004.

8. Gong Qingwu. “The substation simulation training system based on VR technology”. Power grid technology, 2005, 29(24): 74-76.

9. Guan Dandan. "Virtual scene view of ancient ruins” .Xi' an Polytechnic University,2012.

10. Xing Hui. "Design and implementation of remote process control system based on COM technology interface programming”.Qufu Normal University,2006.

11. Du Yuan. "Data real time holographic reconstruction system based on mixed programming of VB and MATLAB” .Kunming University Of Science And Technology,2011.

12. Sun Lu. "Application of immersive virtual environment and Simulation of Substation” .Nanjing University Of Science And Technology,2007. 